\title{
Study on treatment effect of hearing aids on bilateral prolonged tinnitus in Chinese patients
}

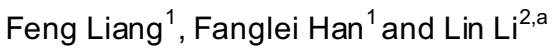 \\ ${ }^{1}$ Department of Anesthesiology, China- Japan Union Hospital, Jilin University, 130033, China \\ ${ }^{2}$ Dept. Otolaryngolgy and head \& Neck, China- Japan Union Hospital, Jilin University, 130033, China
}

\begin{abstract}
Purpose: This study aims to discuss the treatment effect of hearing aids on tinnitus. Methods Sen d questionnaires to 48 cases of bilateral prolonged tinnitus patients with course of more than 1 year to evaluate the subjective perception state of the tinnitus after 24 months. At the same time, follow up 10 cases of bilateral prolonged tinnitus patients without receiving the treatment of hearing aids and use them as the contrast group. Results: Through the treatment of 24 months, the treatment effects on tinnitus are as follows: 23 cases were cured $(47.92 \%), 14$ patients were improved significantly $(29.17 \%), 6$ cases were improved $(12.5 \%)$, and 6 cases were invalid (12.5\%). The overall effective rate was $87.5 \%$.Conclusion: The hearing aids have treatment effect on the bilateral prolonged tinnitus patients in China.
\end{abstract}

\section{Instruction}

The tinnitus usually occurs with deafness and can be a primary central disease (which may be caused by central blood supply efficiency, brain tumor, cranial injury and operation and neurasthenia), and long-term abnormal stimulation for the auditory pathway will also induce a memory in the auditory center to generate long-term tinnitus. The drug treatment effect on the tinnitus is poor. Questionnaires for the bilateral prolonged tinnitus patients who were suggested to wear the hearing aids were used in this study to evaluate the treatment effect of the hearing aids on tinnitus.

\section{Materials and Methods}

\subsection{Patient data}

There were 48 patients who wore the hearing aids for deafness associated with bilateral prolonged tinnitus in Otorhinolaryngology Head and Neck Surgery Department of China-Japan Union Hospital of Jilin University from January to December, 2013, and their courses of disease were more than 12 months. 20 cases were males, 28 cases were females. They were $56-76$ years old and the median age was 68 . The contrast group had 10 cases, with 5 males and 5 females, they were 46-70 years old, and the median age was 65 .

The study was approved by the institutional review board of Ch ina-Japan Union Hospital. All the participants provided written informed consent before the study.

\footnotetext{
${ }^{a}$ Corresponding author: 1ilin01@jlu.edu.cn
} 
The diagnostic assessment of patient with tinnitus should include a case history, audiologic assessment (audiometry, stapedial reflexes, otoacoustic emissions [OAEs]), psychoacoustic tinnitus measures (minimum masking level, loudness, pitch) before trying on the hearing aids. Assessment of tinnitus severity and psychiatric comorbidity (depress ion, anxiety) using standardized questionnaires before the treatment and at the end of two years follow up.

According to the severity of tinnitus and tinnitus with and without concomitant symptoms, the degree of tinnitus was divided into 6 grades ${ }^{[1]}$ : grade 0 : the patient is free of tinnitus; 1 grade: the patient has tinnitus occasionally, but he does not feel pain; grade 2: the patient has prolonged tinnitus, and the tinnitus aggravates when the environment is quiet; grade 3: the patient still has prolong ed tinnitus even in noisy environment; grade 4: the patient has prolonged tinnitus associated with attention and sleep disorders; grade 5: the patient has prolonged severe tinnitus and cannot work properly; grade 6: the patient has the suicidal tendency because of the severe tinnitus. Both ears of each of 48 patients in the treatment group wore the hearing aids.

\subsection{Study de sign}

Digital hearing aids are selected according to the degree of hearing loss, after the patient wears the hearing aids for more than 24 months, subjective changes of the tinnitus are observed, and the grade of tinnitus is assessed according to the grading methods of tinnitus. The patient uses the hearing aids for more than 10 hours every day. After 2 years, the patient were asked whether they accept any other drugs to treat the tinnitus again, and if there was a history of treatment, the patient would withdrawn from the study.

\subsection{Criteria of curative effect and follow-up}

All the 48 patients finish the study, at the end of the 24 months follow-up, all of them filled in a questionnaire to give the result of the treatment and also whether they were satis fied with the result. Curative effect: It is effective if the tinnitus symptom improves to grade 2; it is remarkably effective if the tinnitus symptom improves to grade 2 or above; and the patient is cured if the tinnitus symptom disappears. The patients do not use drugs for tinnitus treatment in this period.

\section{Results}

All the 42 patients finished the study. 42 patients who wore the hearing aids on their two ears clearly stated that the treatment was effective. 23 cases of patients were cured $(47.92 \%), 14$ patients were improved significantly (29.17\%), 6 cases were improved (12.5\%), and 6 cases were invalid (12.5\%). The overall effective rate was $87.5 \%$. While in the contrast group, no patient stated that the tinnitus was relieved. The hearing aids have treatment effect on the tinnitus through the chi-square test $(\mathrm{P}<$ $0.05)$.

Table 1. the effect on the treatment of tinnitus compared with hearing aids group and control group.

\begin{tabular}{|l|l|l|}
\hline & Effective & invalid \\
\hline Hearing aids group $(\mathrm{n}=42)$ & 36 & 6 \\
\hline Control group $(\mathrm{n}=10)$ & 0 & 10 \\
\hline $\mathrm{P}<0.05$ & & \\
\hline
\end{tabular}

\section{Discussions}

Tinnitus is a distressing problem involved in auditory perception, and affects the quality of life of the suffered. Until now, there was no effective or specific drug therapy available, although much research 
work into mechanism and possible treatment is underway. Many clinic studies found that the drug treatment on subjective tinnitus is uncertain [2]. The tinnitus retraining therapy (TRT) was now the suggested treatment for tinnitus ${ }^{[3]}$, the mechanis ms is that TRT could reduce the sensitivity of a central system to the tinnitus until it disappears, to rebuild the filtering function of the auditory system, to stop the auditory feeling on tinnitus and to enable the auditory center to forget the memory for tinnitus gradually. TRT consists of counseling and sound therapy, both based on the neurophysiological model of tinnitus ${ }^{[4]}$. Although TRT is efficacious in most patients, but some patients could not accepted the treatment because of the economical burden and district limited.

The tinnitus happened together with the hearing loss in many cases. Hearing aid was used to improve the hearing level and language reorganization, and nowadays the hearing aid was already accepted by the doctor and patients in China. In some case the tinnitus was found immediately dismissed even when the patient was trying on the hearing aid. But sometimes the patients felt no changing on their tinnitus. But more and more researches pointed out that the treatment of hearing aids may have certain treatment effect on the tinnitus associated with hearing $\operatorname{loss}^{[5]}$, and some methods, for example, both tinnitus handicap inventory (THI) and the international outcome inventory for hearing aids could decrease the tinnitus significantly, both of them improved the patients' satis factions ${ }^{[6]}$. After the patients wore the hearing aids, background noise was amplified properly, and the hearing aids could have the effect of the tinnitus retraining therapy when the surrounding environment was quiet. Our study found that if the patients wore the hearing aids for more than 10 hours each day continuously for a long time ( 2 years), they would have a good treatment effect on most bilateral prolonged tinnitus. In addition, the psychological states of the patients may also be adjusted by the hearing aids, so that the subjective feeling of the tinnitus of the patients can be alleviated ${ }^{[7-8]}$.

In conclusion, in Chinese group, we could use the hearing aid to treat the prolonged tinnitus even the patients who found it was no effect at the beginning of the hearing aids trying. And patient's education might be also important to encourage the patients to have confident on the longtime treatment.

\section{Acknowledgement}

All authors declared no conflict of interest in the research. The work presented in this report is the subject of a pending patent filed by the China-Japan Union Hospital of Jilin University (201510382781.X). This study was supported by the National Natural Science Foundation of China (811007020), the Health and Family Planning Foundation of Jilin Province (20152046), the Science and Technology Development Plan Foundation of Jilin Province (20160101070JC), and the Norman Bethune Program of Jilin University (2015305).

\section{References}

1. Trevis KJ, McLach lan NM, Wilson SJ. Front Psychol. 71262 (eCollection 2016)

2. Michiels S, Van de Heyning P, Truijen S, Hallemans A, De Hertogh W. Man Ther. 26 125-131 (2016)

3. Kim SH, Byun JY, Yeo SG, Park MS. J Int Adv Otol. 12(1) 72-76 (2016)

4. Jastreboff PJ. HNO.63(4) 307-311(2015)

5. Sereda M, Hoare DJ, Nicholson R, Smith S, Hall DA.Ear Hear. 36(4):417-29(2015)

6. Zarenoe R, Söderlund LL, Andersson G Led in T. J A m Acad Audiol. 27(8) 669-76 (2016)

7. Cabral J, Tonocchi R, Ribas Â, Almeida G Rosa M, Massi G Berberian AP. Int Tinnitus J. 20(1) 54-58 (2016)

8. Derek J Hoare, Phillip E Gander, Luke Collins, Sandra Smith, and Deborah A Hall. J Eval Clin Pract. 18(2) 326-334(2012) 